\title{
Craniovertebral junction instability as an extension of cocaine-induced midline destructive lesions: case report
}

\author{
Carlo Brembilla, MD, ${ }^{1}$ Luigi Andrea Lanterna, PhD, ${ }^{1}$ Andrea Risso, MD, ${ }^{1}$ Enrico Bombana, MD, ${ }^{2}$ \\ Paolo Gritti, MD, ${ }^{3}$ Rosangela Trezzi, MD, ${ }^{4}$ Giuseppe Bonaldi, MD, ${ }^{5}$ and Francesco Biroli, MD ${ }^{1}$
}

\begin{abstract}
Departments of ${ }^{1}$ Neurosurgery, ${ }^{2}$ Infectious Diseases, ${ }^{3}$ Anesthesia and Intensive Care, ${ }^{4}$ Pathology, and ${ }^{5}$ Neuroradiology, Pope John XXIII Hospital, Bergamo, Italy
\end{abstract}

\begin{abstract}
With the increasingly widespread illicit use of cocaine, a broad spectrum of clinical pathologies related to this form of drug abuse is emerging. The most frequently used method of administration of powdered cocaine is intranasal inhalation, or "snorting." Consequently, adverse effects of cocaine on the nasal tract are common. Habitual nasal insufflations of cocaine can cause mucosal lesions. If cocaine use becomes chronic and compulsive, progressive damage of the mucosa and perichondrium leads to ischemic necrosis of the septal cartilage and perforation of the nasal septum. Occasionally, cocaine-induced lesions cause extensive destruction of the osteocartilaginous structures of the nose, sinuses, and palate and can mimic other diseases such as tumors, infections, and immunological diseases. In the literature currently available, involvement of the craniovertebral junction in the cocaine-induced midline destructive lesions (CIMDLs) has never been reported.

The present case concerns a 44-year-old man who presented with long-standing symptoms including nasal obstruction, epistaxis, dysphagia, nasal reflux, and severe neck pain. A diagnosis of CIMDL was made in light of the patient's history and the findings on physical and endoscopic examinations, imaging studies, and laboratory testing. Involvement of the craniovertebral junction in the destructive process was evident. For neurosurgical treatment, the authors considered the high grade of atlantoaxial instability, the poorly understood cocaine-induced lesions of the spine and their potential evolution over time, as well as cocaine abusers' poor compliance. The patient underwent posterior craniovertebral fixation. Understanding, classifying, and treating cocaine-induced lesions involving the craniovertebral junction are a challenge. http://thejns.org/doi/abs/10.3171/2014.11.SPINE1471
\end{abstract}

KEY WORDS cocaine-induced midline destructive lesions; craniovertebral junction instability; craniovertebral junction fixation; cervical

I $\mathrm{N}$ Europe, it is estimated that around 13 million adults (15-64 years of age) have used cocaine at least once in their lifetime. ${ }^{5}$ With the increasingly widespread illicit use of cocaine, a broad spectrum of clinical pathologies related to this form of drug abuse is emerging. The most frequently used method of administration of powdered cocaine is intranasal inhalation, or "snorting." 18 Habitual nasal insufflation of cocaine powder often causes considerable damage to nasal mucosa; however, damage of the underlying perichondrium leading to nasal septum perforation and destruction of the osteocartilaginous scaffold of the nose, sinuses, and palate is much less common. ${ }^{25}$ In more severe cases, the destruction extends to the middle and superior turbinates and the lateral wall of the nose. ${ }^{13}$ In some patients, hard and soft palate perforations may be present. Some individuals with severe destruction develop symptoms caused by propagating infections associated with pseudotumor, proptosis, and diplopia. ${ }^{13}$ Midline destructive lesions can be caused by a variety of specific conditions other than cocaine abuse, including infections, neoplasms, sarcoidosis, and granulomatosis with polyangiitis (GPA; Wegener's granulomatosis). Their differentiation is important but may, at times, be very difficult. ${ }^{25}$ The correct diagnosis of cocaine-induced midline destructive lesions (CIMDLs) ultimately depends on a patient's clinical history and documentation of drug abuse combined with the exclusion of other etiologies. ${ }^{15}$ In the currently available literature, involvement of the craniovertebral junction or the spine in general in CIMDLs has never been reported.

ABBREVIATIONS c-ANCA = cytoplasmic antineutrophil cytoplasmic antibody; $\mathrm{CIMDL}=$ cocaine-induced midline destructive lesions; $\mathrm{GPA}=$ granulomatosis with polyangiitis; $\mathrm{HNE}=$ human neutrophil elastase; $\mathrm{p}-\mathrm{ANCA}=$ perinuclear $\mathrm{ANCA}$; VAS = visual analog scale.

SUBMITTED January 22, 2014. ACCEPTED November 12, 2014.

INCLUDE WHEN CITING Published online May 8, 2015; DOI: 10.3171/2014.11.SPINE1471.

DISCLOSURE The authors report no conflict of interest concerning the materials or methods used in this study or the findings specified in this paper. 


\section{Case Report}

History and Examination

A 44-year-old man presented with long-standing symptoms of nasal obstruction, epistaxis, and severe facial pain. Over time he reported progressive worsening of the symptomatology along with dysphagia and nasal reflux. He had originally noticed the problem 2 years earlier, but he came to the attention of Pope John XXIII Hospital in Bergamo, Italy, only after presenting with severe neck pain. He denied any history of trauma but reported a 25-year history of intranasal cocaine abuse. Physical examination on admission revealed severe contracture of the cervical muscles with a severe reduction in craniovertebral junction motion but no neurological signs. The neck pain score on a visual analog scale (VAS) of 0-100, with 0 representing no pain and 100 representing severe pain, was $90 .{ }^{1}$ Nasal endoscopy showed diffuse necrotizing ulcerative lesions of the midline, soft palate, and posterior wall of the rhinopharynx; both a septal and a hard palate perforation; and uvula necrosis.

A complete blood count showed mild normocytic anemia and moderate leukocytosis. Laboratory analysis revealed normal levels of creatinine, electrolytes, serum proteins, and serum angiotensin-converting enzymes and normal liver function tests. Tests for rheumatoid factors as well as cytoplasmic antineutrophil cytoplasmic antibody (c-ANCA), anti-DNA, and antimitochondrial antibodies were done without pathological results.

Computed tomography (Fig. 1) and MRI (Fig. 2) studies confirmed the presence of previous midline lesions as well as cartilaginous reabsorption involving sinonasal structures (septal and hard palate perforations), C1-2 subluxation without myelopathy, and severe bone reabsorption of the odontoid process together with the presence of a paraspinal, epidural, and retropharyngeal thin layer of soft tissue. The retropharyngeal prevertebral layer extended from C-2 to the clivus; it was contrast enhanced and compatible with inflammatory and/or infectious tissue. Dynamic radiographs showed severe atlantoaxial instability (Fig. 3).

Multiple biopsy specimens from necrotizing ulcerative lesions of the soft palate and the posterior wall of the rhinopharynx revealed granulation tissue with mixed inflammatory infiltrates, microabscesses, vascular microthrombotic changes, fibrinoid necrosis, as well as hyphae and spores. A biopsy specimen from the retropharyngeal tissue also revealed inflamed granulation tissue with numerous polymorphs, vascular microthrombotic changes, and hyphae and spores (Fig. 4). Samples for bacterial and fungal cultures from all biopsy sites showed Pseudomonas aeruginosa, Enterococcus faecium, and Candida krusei.

A diagnosis of CIMDL was made in light of the patient's history as well as findings at the physical and endoscopic examinations, imaging studies, and laboratory testing. At the same time, involvement of the craniovertebral junction with atlantoaxial instability was evident in the destructive process. The neuroradiological examinations showed-besides the atlantoaxial instability-extended bone reabsorption and a paraspinal, epidural, and retropharyngeal thin layer of soft tissue. Extended bone
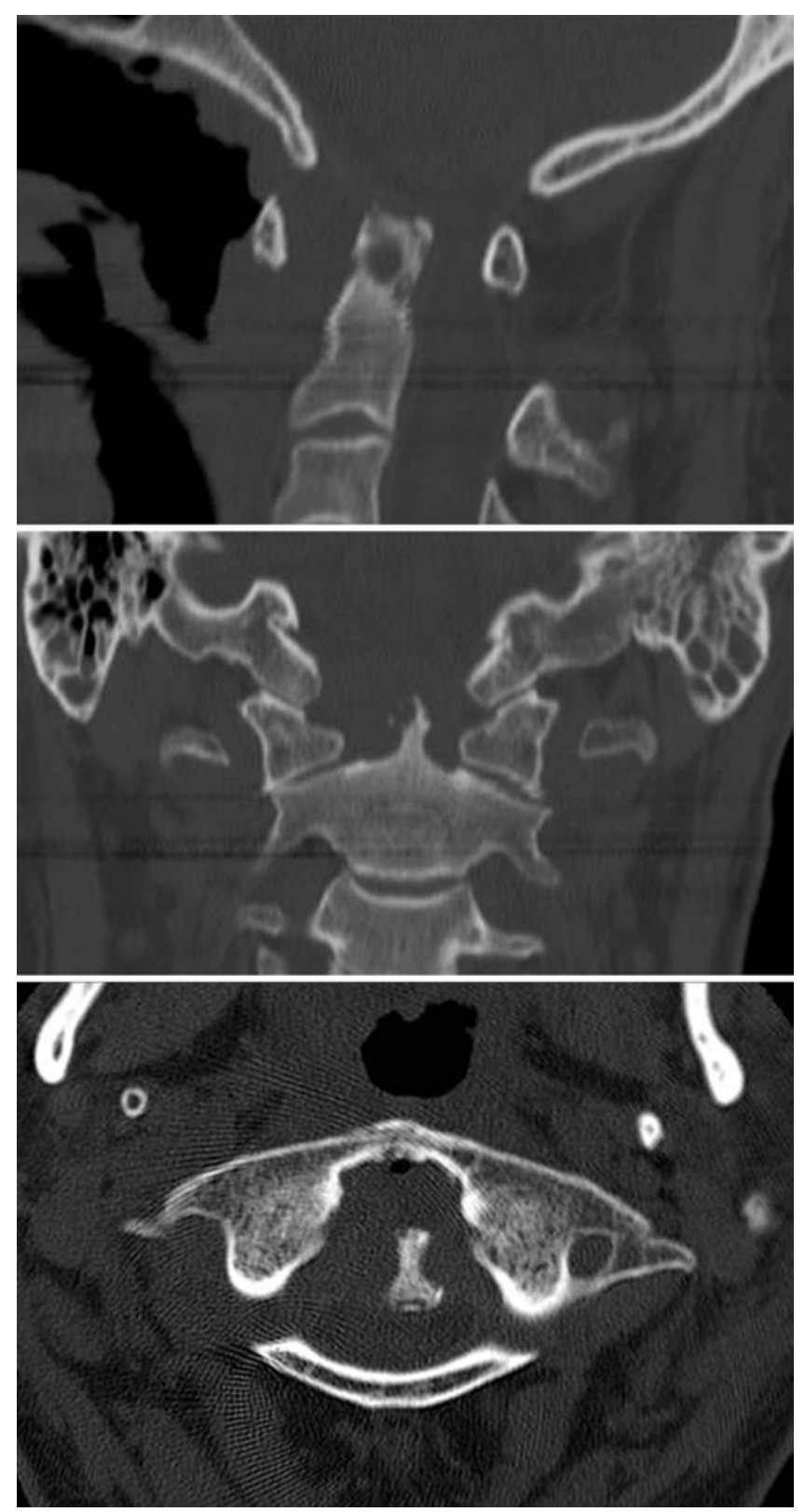

FIG. 1. Computed tomography scans showing C1-2 subluxation with severe bone reabsorption of the odontoid process.

reabsorption is characteristic in CIMDL, and the biopsy specimen from the retropharyngeal layer of soft tissue had the same characteristics as the biopsy specimens from the necrotizing ulcerative lesions of the soft palate and posterior wall of the rhinopharynx. The patient denied any history of trauma that may have caused the atlantoaxial instability. There was no evidence of other pathologies. Superinfections from all the biopsy sites were the same. All of these findings led us to consider the craniovertebral junction pathology as an extension of the CIMDL.

\section{Treatment}

The patient was informed that any chance of therapeutic success was entirely dependent on continued absti- 

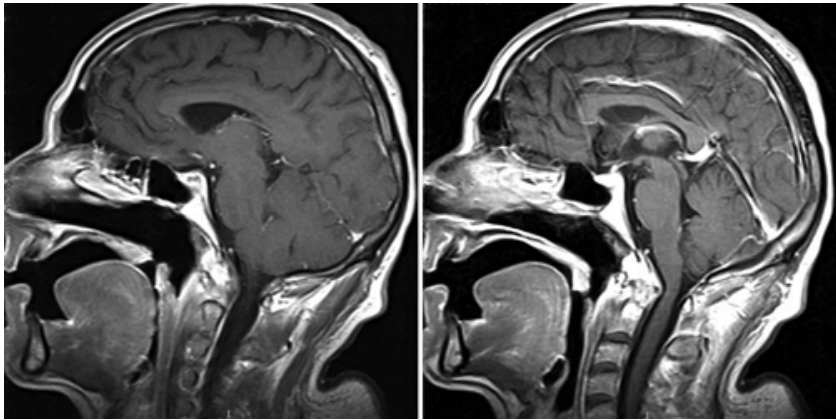

FIG. 2. Magnetic resonance images showing $\mathrm{C} 1-2$ subluxation without myelopathy; the presence of a paraspinal, epidural, and retropharyngeal layer of soft tissue extending from $\mathrm{C}-2$ to the clivus compatible with inflammatory and/or infectious tissue; soft palate and posterior wall of the rhinopharynx perforations; large nasal septum and turbinates reabsorption.

nence from cocaine. The uvula was removed endoscopically. Conservative local treatments such as careful debridement of necrotic tissues and crusts and regular saline douches were started. Surgical correction of the septal and hard palate perforations was deferred until 6 months after the cessation of cocaine abuse, and toxicology tests were performed to verify that the patient refrained from taking cocaine. He started systemic antibiotic therapy with meropenem trihydrate and daptomycin and antifungal therapy with fluconazole.

As regards neurosurgical treatment, most importantly we considered that craniovertebral instability correlated with CIMDL had never been described, so its potential evolution over time was unknown. Currently, both CIMDL and cocaine-induced craniovertebral instability are poorly understood. Neuroradiological examination showed instability at the $\mathrm{C} 1-2$ articulation (not at the $\mathrm{C} 0-1$ articulation), but also a retropharyngeal prevertebral layer compatible with inflammatory and infectious tissue and extending from $\mathrm{C}-2$ to the clivus (involving the $\mathrm{C} 0-1$ articulation). Cocaine abusers' poor compliance in terms of abstinence from cocaine and follow-up behavior was considered in the treatment planning. After all of these con-

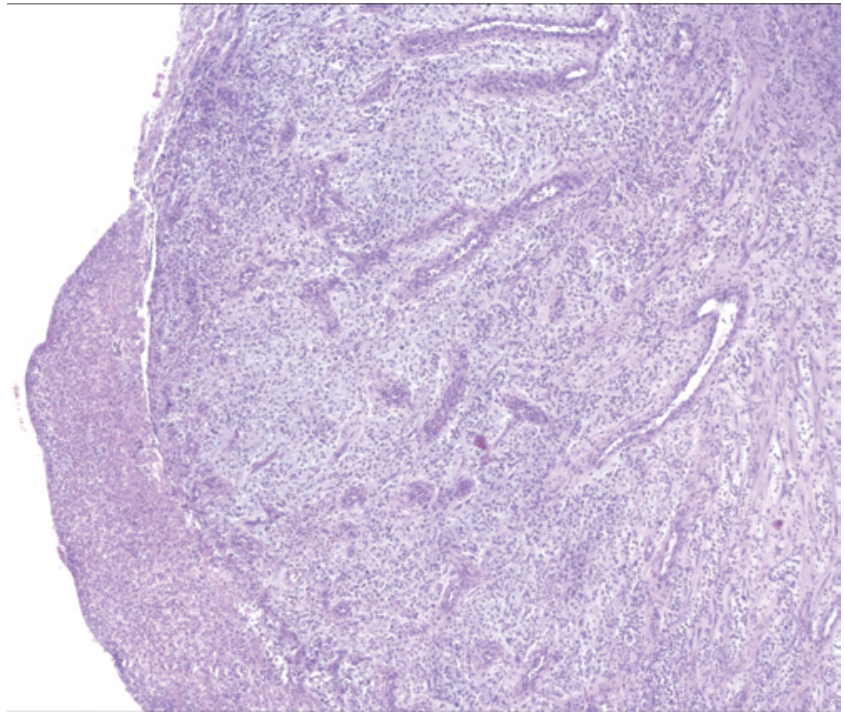

FIG. 4. Ulcerated, inflamed granulation tissue from the retropharyngeal wall with numerous polymorphs and vessels of varying sizes within loose connective tissue. $\mathrm{H} \& \mathrm{E}$, original magnification $\times 200$. Figure is available in color online only.

siderations, a more aggressive craniovertebral fixation was preferred, instead of atlantoaxial stabilization and fusion that could preserve $\mathrm{C} 0-1$ motion, to prevent progression of the instability at the $\mathrm{C} 0-1$ articulation over time. The patient underwent an aggressive posterior craniovertebral fixation after a reduction in $\mathrm{C} 1-2$. The reduction was intraoperatively performed with $\mathrm{C}-1$ posterior wiring: after rod positioning, 2 braided nonabsorbable surgical sutures (Ethibond Excel Polyester Suture, Ethicon) were tensioned between the $\mathrm{C}-1$ posterior arch with one suture on each rod and fixed. A C1-2 intraarticular fusion was provided with the apposition of demineralized bone matrix (DBX, Synthes) after decortication of the facet surfaces. Note that $\mathrm{C} 0-1$ was not fused (it was not considered unstable) but was only stabilized to prevent the development of any possible future cocaine-induced craniovertebral lesions.
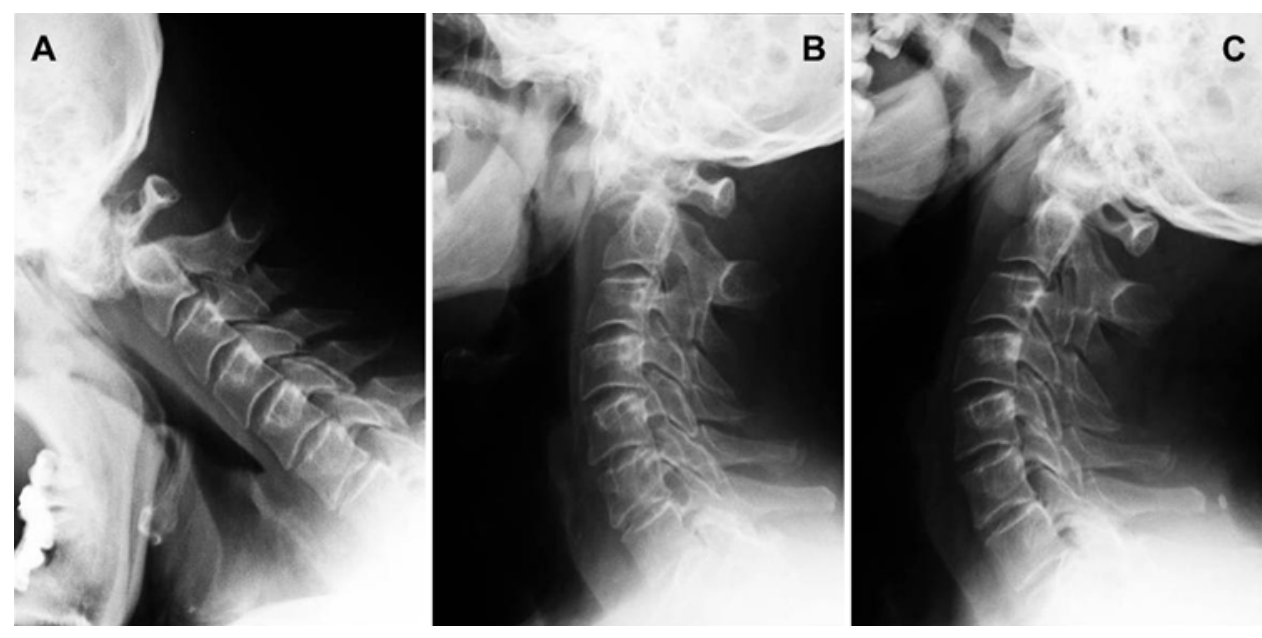

FIG. 3. Dynamic flexion (A), neutral position (B), and extension (C) radiographs showing severe atlantoaxial instability. 


\section{Posttreatment Course}

The patient walked with a cervical brace for 1 month and was referred to the physical medicine departments for proper rehabilitation therapy. On postoperative Day 1, 2 plain radiographic scans were obtained. Two plain cervical radiographs were also obtained at 4 weeks and 3 and 6 months after surgery to assess the stability of the implant, correct alignment of the levels treated, and the rate of fusion.

After 6 months, the patient reported no neck pain, with a 0 score on the VAS and no neurological signs. A radiographic check showed good alignment of the craniovertebral junction, stability of the fixation, and $\mathrm{C} 1-2$ interarticular fusion (Fig. 5). Endoscopy showed that the midline lesions were improved but still present; no other lesions were found on the posterior wall of the rhinopharynx. Samples for bacterial and fungal cultures demonstrated no infections. The patient's abstinence from cocaine was inconsistent, with occasional use. Surgical correction of the septal and hard palate perforations was still deferred until at least 6 months of complete abstinence had been achieved.

After the 6-month check-up, the patient failed to attend the follow-up program. He returned to the attention of our institution after 11 months (1 year 5 months after the surgery) under detention. He reported no neck pain, with a 0 score on the VAS and no neurological signs. Dynamic radiographs showed good alignment of the craniovertebral junction, stability of the fixation, and C1-2 interarticular fusion (Fig. 6). Magnetic resonance imaging showed good alignment of the craniovertebral junction; involution of the craniovertebral lesion with reabsorption of the paraspinal, epidural, and retropharyngeal layer of soft tissue compatible with inflammatory and/or infectious tissue; soft and hard palate perforations (no further perforation of the posterior wall of the rhinopharynx); and large nasal septum and turbinate reabsorption (Fig. 7). Endoscopy showed that the midline lesions were stable with respect to the last check-up. Samples for bacterial and fungal cultures demonstrated no infections. Abstinence from cocaine was still incomplete, with occasional use, as is often the case in patients with a long-standing drug addiction history. The patient reported moderate but well-managed dysphagia and nasal reflux. Considering the clinical situation and the drug addiction, surgical correction of the septal and hard palate perforations was not performed or even planned. A 6-month follow-up was scheduled.

\section{Discussion}

The European commission on drug abuse has stated that 13 million adults (15-64 years) have used cocaine at least once in their lifetime, and some countries (Spain, the United Kingdom, Denmark, Ireland, Italy) have been identified with a higher prevalence of use among young adults (15-34 years). ${ }^{5}$ The National Survey on Drug Use and Health reported that in 2008, cocaine was used by 36.7 million Americans. ${ }^{19}$ The illicit use of cocaine means a wider spectrum of clinical pathologies related to this form of drug abuse. ${ }^{18}$ As nasal insufflation is the most frequently used route of administration, adverse effects to the

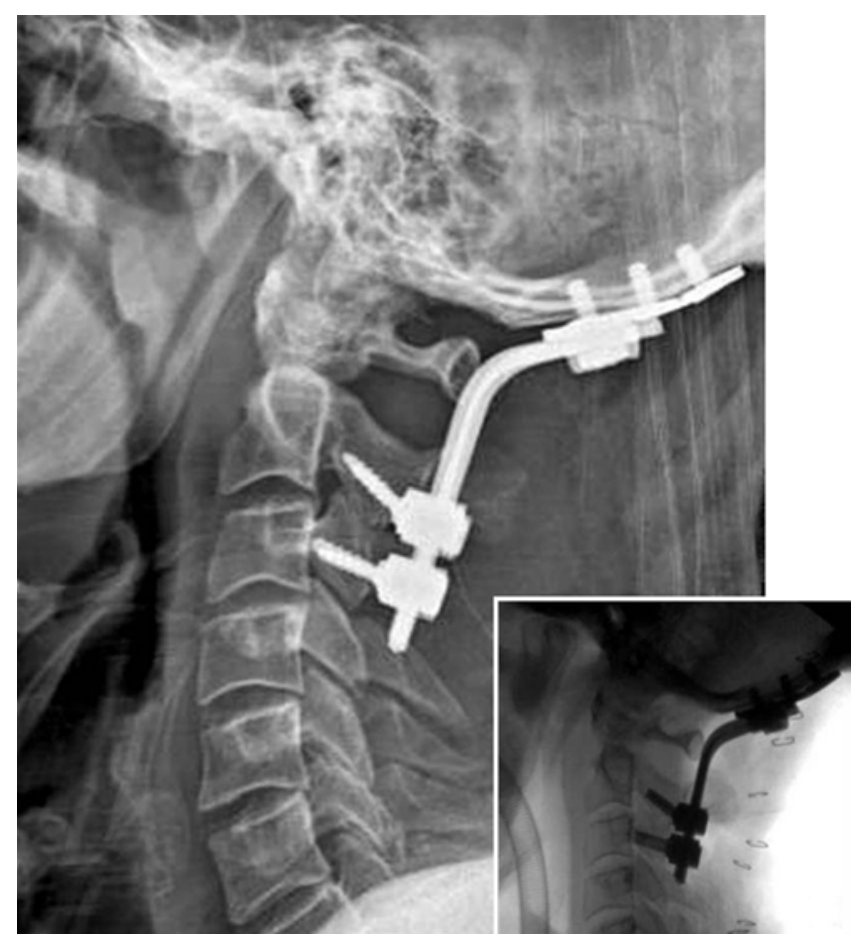

FIG. 5. Intraoperative fluoroscopic image and 6-month postoperative craniovertebral spine radiograph (inset). The latter showed good alignment of the craniovertebral junction, stability of the fixation, and C1-2 interarticular fusion.

nasal tract are common. ${ }^{15}$ Habitual insufflation of cocaine powder often causes considerable damage to nasal mucosa and the underlying perichondrium.

So-called CIMDLs display a typical clinical pattern. Long-standing symptoms include nasal obstruction, epistaxis, and severe facial pain. The most common findings are diffuse necrotizing ulcerative lesions, extensive crusting, and septal perforation. ${ }^{13}$ In more severe cases, the destruction extends to the middle and superior turbinates and the lateral wall of the nose. ${ }^{13}$ In some patients, hard and soft palate perforations may be present and cause dysphagia and nasal reflux and substantially affect quality of life..$^{13}$ Some individuals with severe destruction develop symptoms caused by propagating infections associated with pseudotumor, proptosis, and diplopia. ${ }^{13}$ Symptoms such as fever, malaise, weight loss, as well as arthralgia or myalgia, are typically absent, and patients with CIMDL usually do not have any symptoms or laboratory findings indicating a systemic disease at presentation. ${ }^{13}$

Magnetic resonance imaging represents the most useful first-line choice for radiological evaluation of patients with CIMDL. ${ }^{23,24}$ Magnetic resonance imaging can detect areas of abnormal nasal or paranasal mucosa. ${ }^{11,25}$ In cocaine abusers, significant enlargement of the palatine or pharyngeal tonsils has been associated with small fluid collections within lymphatic tissue. ${ }^{23}$ Radiographic signs of otitis media may also be detected. In some patients, progressive "centrifugal" involvement of the lateral nasal walls and floor with eventual destruction of the entire framework and complete erosion of the hard and soft palate have been noted. ${ }^{25}$ Computed tomography scans are 

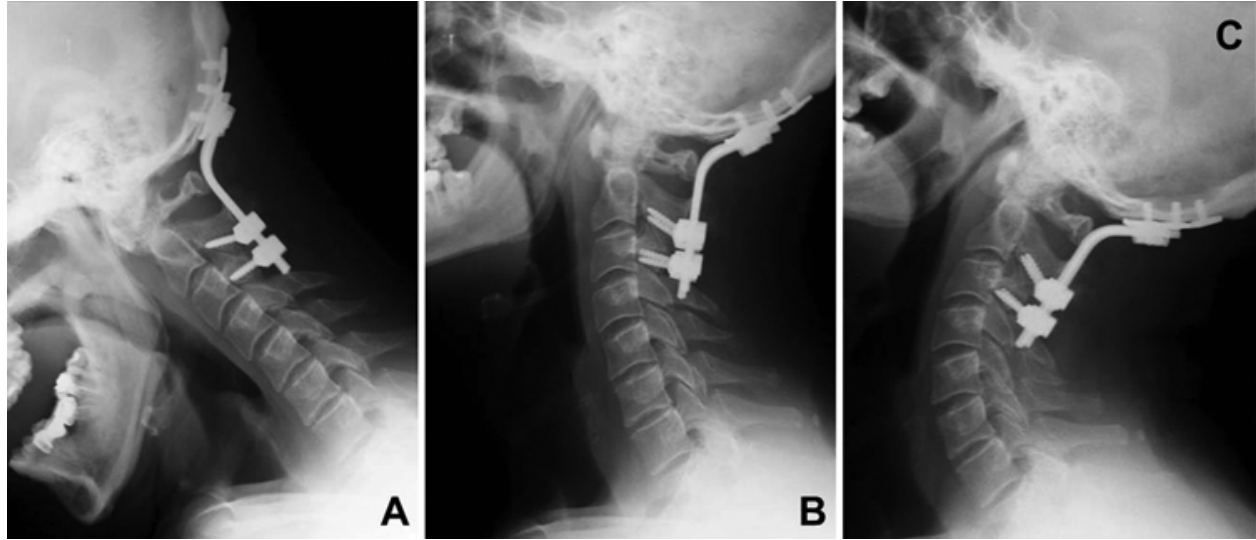

FIG. 6. Dynamic radiographic images in flexion (A), neutral position (B), and extension (C) obtained at 1 year 5 months after surgery, showing good alignment of the craniovertebral junction, stability of the fixation, and C1-2 interarticular fusion.

used to determine the presence of mucosal inflammatory lesions as well as cartilaginous and/or bony reabsorption involving sinonasal structures. ${ }^{15}$

Midline destructive lesions can be caused by a variety of specific conditions other than cocaine abuse, including infections, neoplasms, sarcoidosis, and GPA (Wegener's). Their differentiation is important but may at times be very difficult. ${ }^{25}$ The correct diagnosis of CIMDL depends on the patient's clinical history and documentation of drug abuse combined with the exclusion of other etiologies. ${ }^{15}$ Multiple biopsy specimens and samples for bacterial and fungal cultures should also be obtained. Infections such as tuberculosis, tertiary syphilis, leishmaniasis, mucormycosis, or infections caused by other organisms can also lead to septal perforation or osteocartilaginous destruction in the nasal cavity. The histopathological identification of sarcoidosis or lymphoproliferative disorders as the cause of midline destructive lesions is usually straightforward. However, differentiation of CIMDL from limited forms of GPA can be more difficult. ${ }^{23-25}$ Histopathological features shared between CIMDL and GPA-and thus not allow- ing for their differentiation-include mixed inflammatory infiltrates, microabscesses in vascular walls, perivenulitis, vascular microthrombotic changes, leukocytoclastic vasculitis, and fibrinoid necrosis. ${ }^{23}$ Only extravascular changes such as stromal granulomas with giant cells, microabscesses, and deeply located necrosis are features that seem to be pathognomonic for GPA. ${ }^{23}$ Only if these specific features are found in the biopsy specimen can GPA be histopathologically diagnosed, and CIMDL is unlikely. In the absence of these features, histopathological differentiation between CIMDL and GPA is impossible. In contrast, serological differentiation between CIMDL and GPA is less difficult. Almost all patients with CIMDL have circulating serum antineutrophil cytoplasmic antibodies (ANCAs). ${ }^{27}$ In patients with CIMDL, these ANCAs are primarily directed against human neutrophil elastase (HNE), generate a perinuclear (p-ANCA) staining pattern on ethanol-fixed neutrophils, and do not react with myeloperoxidase. ${ }^{27}$ In contrast, patients with GPA frequently have ANCAs that generate a cytoplasmic (c-ANCA) staining pattern on ethanol-fixed neutrophils that reacts with proteinase 3. Con-

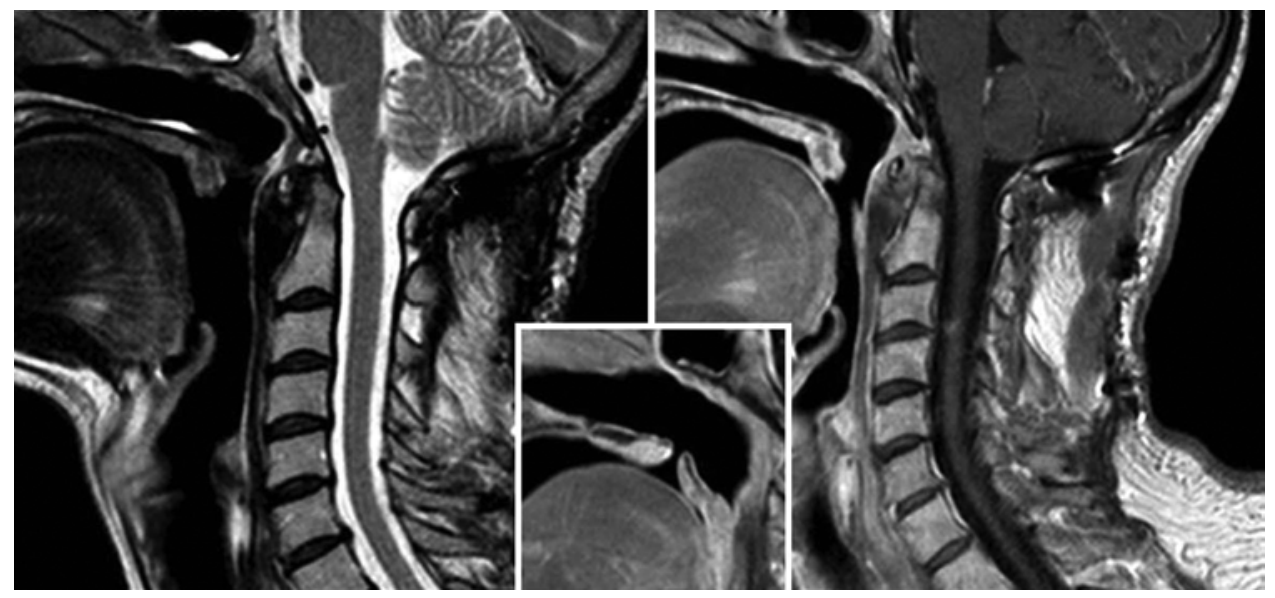

FIG. 7. Magnetic resonance images obtained 1 year 5 months after surgery, showing good alignment of the craniovertebral junction; reabsorption of the paraspinal, epidural, and retropharyngeal layer of soft tissue compatible with inflammatory and/or infectious tissue; soft and hard palate perforations (no further perforation of the posterior wall of the rhinopharynx); and large nasal septum and turbinate reabsorption. 
sequently, in clinical practice, both immunofluorescence and antigen-specific solid-phase assay testing need to be performed in parallel when differentiation of CIMDL from GPA is sought. ${ }^{14-22}$

The pathogenesis of CIMDL remains poorly understood. Vascular ischemia from excessive cocaine use is widely considered to be a major element in the development of CIMDL..$^{3-8}$ However, given the widespread abuse of nasal cocaine insufflation on the one hand and the relative rarity of CIMDL on the other, differentiating host factors must hold the key to the undoubtedly multifactorial pathogenesis of CIMDL. ${ }^{20}$ The ischemic and direct traumatic effects of cocaine crystals should affect all users similarly. ${ }^{17}$ The strong tendency of the damaged nasal mucosa to form tightly adhering scabs prompts affected patients to remove these scabs, often by using further damage-inducing mechanical methods ${ }^{16}$ Frequency, severity, and tenacity of bacterial superinfections of the damaged nasal mucosa may be related to the severity of the underlying mucosal damage as well as to individual nasal hygiene and antimicrobial treatment patterns. Bacterial superinfection has been documented in essentially all patients with CIMDL. ${ }^{23}$ What seems to distinguish patients with CIMDL from individuals with similar cocaine use patterns without CIMDL is the presence of ANCA in CIMDL. ${ }^{27}$ Bacterial infections with superantigen-producing organisms may lead to ANCA production and longterm persistence in individuals predisposed to autoimmunity. ${ }^{21}$ The predominant ANCA type in CIMDL is directed against HNE. ${ }^{27}$ The presence of HNE-ANCA may significantly enhance the local inflammatory response to injury. Moreover, cocaine seems to induce apoptosis of the respiratory epithelial cells and perhaps of the inflammatory cells in the nasal passages. ${ }^{24}$ The apoptosis-inducing effects of cocaine are dose and time dependent. ${ }^{24}$ In summary, CIMDLs seem to be the result of a necrotizing inflammatory tissue response triggered by cocaine abuse in a subset of patients predisposed to produce ANCA, particularly those reacting with HNE. ${ }^{22}$

The management of cocaine abusers represents a real clinical challenge. There is no role for immunosuppressive therapy in the management of CIMDL, and patients need to understand that any chance of therapeutic success is entirely dependent on continued abstinence from cocaine. Medical therapy, prosthesis, or surgery can lead to good results if patients reliably stop snorting. ${ }^{25}$ Unfortunately, cocaine abusers are notorious for their poor compliance and dishonesty with physicians. ${ }^{25}$ In patients in whom lesions occur very early, the discontinuation of drug use may lead to gradual normalization of the nasal mucosa and reversal of the pathological process. ${ }^{25}$ Conservative local treatments such as careful debridement of necrotic tissues and crusts, regular saline douches, and local or systemic application of antibiotic therapy are recommended. ${ }^{25}$ An obturator prosthesis for palate defects can overcome the oronasal reflux, ${ }^{25}$ but surgical correction of mucosal or cutaneous defects should be postponed until a lesion is stable. The decision to treat (or not), as well as the best timing of treatment in relation to the duration of abstinence, represents a challenge. The minimum time interval free of cocaine use remains controversial, and there is no con- sensus or large body of experience to provide guidance. Some authors ${ }^{4-26}$ insist on the need to confirm the cessation of cocaine abuse with toxicological tests, ensuring full rehabilitation for 6 or even 12 months prior to surgery, ${ }^{7-10}$ to avoid treatment failure. The surgical procedures most often requested by patients are septal and palate perforation closure. ${ }^{2-9}$ However, some patients ask for aesthetic procedures such as rhinoplasty and closure of nasocutaneous fistulas. ${ }^{6,12,26}$

In the literature currently available, involvement of the craniovertebral junction or the spine in general in CIMDL has never been reported. The featured case is only observational and the follow-up was short: 1 year, 5 months. More cases must be observed to confirm spinal involvement in CIMDL. If other cases do, in fact, exist, understanding, classifying, and treating these kinds of cocaineinduced lesions involving the craniovertebral junction will present a challenge.

\section{References}

1. Bijur PE, Silver W, Gallagher EJ: Reliability of the visual analog scale for measurement of acute pain. Acad Emerg Med 8:1153-1157, 2001

2. Businco LD, Lauriello M, Marsico C, Corbisiero A, Cipriani O, Tirelli GC: Psychological aspects and treatment of patients with nasal septal perforation due to cocaine inhalation. Acta Otorhinolaryngol Ital 28:247-251, 2008

3. Caravaca A, Casas F, Mochón A, De Luna A, San Martín A, Ruiz A: [Centrofacial necrosis secondary to cocaine use.] Acta Otorrinolaringol Esp 50:414-416, 1999 (Span)

4. Di Cosola M, Turco M, Acero J, Navarro-Vila C, Cortelazzi $\mathrm{R}$ : Cocaine-related syndrome and palatal reconstruction: report of a series of cases. Int J Oral Maxillofac Surg 36:721-727, 2007

5. European Commission: Flash Eurobarometer 233: Young people and drugs among 15-24 year-olds. Analytical report. Brussels: European Commission, 2008

6. Guyuron B, Afrooz PN: Correction of cocaine-related nasal defects. Plast Reconstr Surg 121:1015-1023, 2008

7. Kuriloff DB: Re: Perforation of the hard palate associated with cocaine abuse. Ann Plast Surg 28:397, 1992

8. Kuriloff DB, Kimmelman CP: Osteocartilaginous necrosis of the sinonasal tract following cocaine abuse. Laryngoscope 99:918-924, 1989

9. Ladner T, Linker M, Gitani J: [Functional repair of a major necrotic palatine defect caused by chronic cocaine inhalation.] Rev Stomatol Chir Maxillofac 105:291-293, 2004 (Fr)

10. Lancaster J, Belloso A, Wilson CA, McCormick M: Rare case of naso-oral fistula with extensive osteocartilaginous necrosis secondary to cocaine abuse: review of otorhinolaryngological presentations in cocaine addicts. J Laryngol Otol 114:630-633, 2000

11. Lloyd G, Lund VJ, Beale T, Howard D: Rhinologic changes in Wegener's granulomatosis. J Laryngol Otol 116:565-569, 2002

12. Millard DR, Mejia FA: Reconstruction of the nose damaged by cocaine. Plast Reconstr Surg 107:419-424, 2001

13. Morassi ML, Trimarchi M, Nicolai P, Gregorini G, Maroldi $\mathrm{R}$, Specks U, et al: [Cocaine, ANCA, and Wegener's granulomatosis.] Pathologica 93:581-583, 2001 (Ital)

14. Peikert T, Finkielman JD, Hummel AM, McKenney ME, Gregorini G, Trimarchi M, et al: Functional characterization of antineutrophil cytoplasmic antibodies in patients with cocaine-induced midline destructive lesions. Arthritis Rheum 58:1546-1551, 2008 
15. Seyer BA, Grist W, Muller S: Aggressive destructive midfacial lesion from cocaine abuse. Oral Surg Oral Med Oral Pathol Oral Radiol Endod 94:465-470, 2002

16. Silvestre FJ, Perez-Herbera A, Puente-Sandoval A, Bagán JV: Hard palate perforation in cocaine abusers: a systematic review. Clin Oral Investig 14:621-628, 2010

17. Sittel C, Eckel HE: Nasal cocaine abuse presenting as a central facial destructive granuloma. Eur Arch Otorhinolaryngol 255:446-447, 1998

18. Specks U: The growing complexity of the pathology associated with cocaine use. J Clin Rheumatol 17:167-168, 2011

19. Substance Abuse and Mental Health Services Administration: Illicit Drug Use, in Results from the 2007 National Survey on Drug Use and Health: National Findings. Rockville: SAMHSA, Office of Applied Studies, 2007, pp $15-29$

20. Substance Abuse and Mental Health Services Administration: National Household Survey on Drug Abuse. Rockville: SAMHSA, Office of Applied Studies, 1998

21. Tervaert JW, Popa ER, Bos NA: The role of superantigens in vasculitis. Curr Opin Rheumatol 11:24-33, 1999

22. Trimarchi M, Bussi M, Sinico RA, Meroni P, Specks U: Cocaine-induced midline destructive lesions-An autoimmune disease? Autoimmun Rev 12:496-500, 2013

23. Trimarchi M, Gregorini G, Facchetti F, Morassi ML, Manfredini C, Maroldi R, et al: Cocaine-induced midline destructive lesions: clinical, radiographic, histopathologic, and serologic features and their differentiation from Wegener granulomatosis. Medicine (Baltimore) 80:391-404, 2001

24. Trimarchi M, Miluzio A, Nicolai P, Morassi ML, Bussi M, Marchisio PC: Massive apoptosis erodes nasal mucosa of cocaine abusers. Am J Rhinol 20:160-164, 2006
25. Trimarchi M, Nicolai P, Lombardi D, Facchetti F, Morassi ML, Maroldi R, et al: Sinonasal osteocartilaginous necrosis in cocaine abusers: experience in 25 patients. Am J Rhinol 17:33-43, 2003

26. Vilela RJ, Langford C, McCullagh L, Kass ES: Cocaineinduced oronasal fistulas with external nasal erosion but without palate involvement. Ear Nose Throat J 81:562-563, 2002

27. Wiesner O, Russell KA, Lee AS, Jenne DE, Trimarchi M, Gregorini G, et al: Antineutrophil cytoplasmic antibodies reacting with human neutrophil elastase as a diagnostic marker for cocaine-induced midline destructive lesions but not autoimmune vasculitis. Arthritis Rheum 50:2954-2965, 2004

\section{Author Contributions}

Conception and design: Brembilla, Lanterna. Acquisition of data: Risso, Bombana, Gritti, Trezzi. Analysis and interpretation of data: Trezzi. Drafting the article: Brembilla, Bombana. Critically revising the article: Brembilla, Lanterna, Gritti, Bonaldi. Reviewed submitted version of manuscript: Brembilla, Lanterna, Risso, Bombana, Gritti, Bonaldi, Biroli. Approved the final version of the manuscript on behalf of all authors: Brembilla. Study supervision: Brembilla, Lanterna, Biroli.

\section{Correspondence}

Carlo Brembilla, Department of Neurosurgery, Pope John XXIII Hospital, WHO square no. 1, Bergamo 24100, Italy. email: carlinobrembo@hotmail.com. 